\title{
openheart Major bleeding after percutaneous coronary intervention and risk of subsequent mortality: a systematic review and meta-analysis
}

\author{
Chun Shing Kwok, ${ }^{1}$ Sunil V Rao, ${ }^{2}$ Phyo K Myint, ${ }^{3}$ Bernard Keavney, ${ }^{1}$ \\ James Nolan, ${ }^{4}$ Peter F Ludman, ${ }^{5}$ Mark A de Belder, ${ }^{6}$ Yoon K Loke, ${ }^{7}$ \\ Mamas A Mamas ${ }^{1}$
}

To cite: Kwok CS, Rao SV, Myint PK, et al. Major bleeding after percutaneous coronary intervention and risk of subsequent mortality: a systematic review and meta-analysis. Open Heart 2014;: :e00021. doi:10.1136/openhrt-2013000021

- Additional material is published online only. To view please visit the journal online (http://dx.doi.org/10. 1136/openhrt-2013-000021).

Received 13 December 2013 Revised 11 January 2014 Accepted 18 January 2014

CrossMark

For numbered affiliations see end of article.

Correspondence to Dr Mamas A Mamas; mamasmamas1@yahoo.co.uk

\section{ABSTRACT}

Objectives: To examine the relationship between periprocedural bleeding complications and major adverse cardiovascular events (MACEs) and mortality outcomes following percutaneous coronary intervention $(\mathrm{PCl})$ and study differences in the prognostic impact of different bleeding definitions. Methods: We conducted a systematic review and meta-analysis of $\mathrm{PCl}$ studies that evaluated periprocedural bleeding complications and their impact on MACEs and mortality outcomes. A systematic search of MEDLINE and EMBASE was conducted to identify relevant studies. Data from relevant studies were extracted and random effects meta-analysis was used to estimate the risk of adverse outcomes with periprocedural bleeding. Statistical heterogeneity was assessed by considering the $\mathrm{I}^{2}$ statistic.

Results: 42 relevant studies were identified including 533333 patients. Meta-analysis demonstrated that periprocedural major bleeding complications was independently associated with increased risk of mortality (OR 3.31 (2.86 to 3.82), $I^{2}=80 \%$ ) and MACEs (OR 3.89 (3.26 to 4.64 ), $\left.\right|^{2}=42 \%$ ). A differential impact of major bleeding as defined by different bleeding definitions on mortality outcomes was observed, in which the REPLACE-2 (OR 6.69, 95\% Cl 2.26 to 19.81), STEEPLE (OR 6.59, $95 \% \mathrm{Cl} 3.89$ to 11.16 ) and BARC (OR 5.40, $95 \% \mathrm{Cl} 1.74$ to 16.74 ) had the worst prognostic impacts while HORIZONS-AMI (OR 1.51, 95\% Cl 1.11 to 2.05) had the least impact on mortality outcomes.

Conclusions: Major bleeding after $\mathrm{PCl}$ is independently associated with a threefold increase in mortality and MACEs outcomes. Different contemporary bleeding definitions have differential impacts on mortality outcomes, with 1.5-6.7-fold increases in mortality observed depending on the definition of major bleeding used.

\section{INTRODUCTION}

Advances in antithrombotic therapy have improved the outcomes of patients undergoing

\section{KEY MESSAGES}

- The strength of this systematic review was the large number of studies included with over half a million total participants.

- Another strength was that we were able to evaluate the effect of different major bleeding definitions and its impact on risk of mortality and major adverse cardiovascular events.

- This systematic review had the limitation that studies included varied in antithrombotic and antiplatelet regimes after $\mathrm{PCl}$ procedure.

- Another limitation was that the systematic review was unable evaluate whether the subsequent mortality was directly related to the bleed.

percutaneous coronary intervention (PCI) through the reduction of ischaemic events at the expense of increased procedure-related bleeding complications. Major bleeding events in contemporary PCI are significant, with 30-day bleeding event rates reported between $0.7 \%$ and $1.1 \%$ in elective, ${ }^{1-3} 0.6 \%$ and $4.7 \%{ }^{3-6}$ in non-ST-elevation myocardial infarction (NSTEMI) and $0.9 \%$ and $8.9 \%$ in ST-elevation myocardial infarction (STEMI) ${ }^{3} 478$ depending on the definition used.

There are currently around 10 different definitions of major bleeding used in trials and registries of patients undergoing $\mathrm{PCI}^{9} 10$ and these definitions include various clinical events, such as blood transfusion or retroperitoneal haemorrhage, laboratory parameters, such as differing values of haemoglobin decreases, and clinical outcomes such as mortality ${ }^{9}$ resulting in significant differences in bleeding event recording across clinical trials thereby making comparisons between therapeutic strategies difficult. Furthermore, the incidence of major bleeding varies depending on definition used. In 
one study, the RIVAL non-coronary artery bypass graft (non-CABG) related major bleeding occurred in $0.87 \%$ in the STEMI cohort and $0.57 \%$ in the NSTEMI group, while if an ACUITY major bleeding definition was used, major bleeding occurred in $3.1 \%$ in the STEMI group and $2.26 \%$ in the NSTEMI group, respectively.

Major periprocedural bleeding complications following PCI are predictors of mortality and major adverse cardiovascular events (MACEs), ${ }^{11-13}$ with up to $12.1 \%$ of all in-hospital mortality after PCI in the National Cardiovascular Data Registry's CathPCI Registry related to bleeding complications. ${ }^{14}$ In contrast, other studies have suggested that although bleeding may be causally related to adverse outcomes in some patients in the realworld setting, it is often merely a marker for patients at higher risk for adverse outcomes. ${ }^{15} 16$

Some prior studies that have reported on the prognostic impact of major bleeds have not accounted for differences in baseline covariates such as age, syndrome of presentation and comorbidities that would themselves impact on MACEs and mortality outcomes. ${ }^{17-21}$ In contrast, while other studies have adjusted for baseline covariates, different definitions of major bleeding such as TIMI, ${ }^{2} 1122{ }^{23}$ GUSTO $^{20}{ }^{24}$ STEEPLE $^{20}{ }^{25}$ and BARC $^{23}$ have been used, which have been shown to have differential impacts on mortality/MACEs outcomes..$^{20} 2326$ Furthermore, the timing of bleeding from index PCI procedures included in such studies has varied from $48 \mathrm{~h},{ }^{25}$ been limited to those that occur in hospital, ${ }^{23} 27$ to 30 days $^{2}{ }^{28}$ with impact on mortality and MACEs outcomes studied at different time points such as 30 days, ${ }^{82428} 6$ months $^{2427}$ or 1 year. ${ }^{2} 222325$

Until today, there has not been a systematic review or meta-analysis previously published studying the prognostic impact of periprocedural major bleeding events on mortality and MACEs outcomes following PCI. We have therefore undertaken a meta-analysis to systematically study the impact of major bleeding following PCI on mortality and MACEs outcomes. In this meta-analysis, we provide an overview of the cohorts evaluating the rates of major bleeding events and systematically study the differences in the prognostic impact of different bleeding definitions and the relationship between major bleeding and clinical events at different time points.

\section{METHODS}

\section{Eligibility criteria}

We selected studies of patients who underwent PCI that reported on mortality or cardiovascular events among patients with and without major bleeding events. There was no restriction based on study design, definition of major bleeding or the indication for PCI or its status as an urgent or elective procedure. We excluded studies that did not report on categories of major bleeding and those that did not report either mortality or MACEs.

\section{Search strategy}

A search of EMBASE (1974 to January 2014) and MEDLINE (1946 to January 2014) was conducted on OvidSP. The search terms are shown in online supplementary figure S1. We did not use any language restrictions. We checked the bibliographies of included studies and relevant review articles found on the search for additional relevant articles.

\section{Study selection and data extraction}

Two reviewers (CSK and YKL) checked all titles and abstracts for studies that could potentially meet the inclusion criteria. We retrieved full reports of these potentially eligible studies and independently extracted data on study design, participant characteristics, interventions used, major bleeding events, follow-up, outcome events and methods of ascertaining measured clinical events on to a preformatted spreadsheet. Any discrepancies between the two reviewers were resolved by consensus after consulting a third reviewer (MAM).

\section{Quality assessment}

Risk of bias was assessed by considering four different areas: ascertainment of major bleeding events, ascertainment of outcome events, extent of loss to follow-up and the use of adjustment for confounders in the analysis. We also assess for publication bias using funnel plots when there were $>10$ studies available in the meta-analysis and there was no evidence of substantial statistical heterogeneity. ${ }^{29}$

\section{Data analysis}

We used RevMan V.5.1.7 (Nordic Cochrane Centre) to do random effects meta-analysis using the inverse variance methods for pooled ORs. We assumed similarity between the OR and other relative measures such as relative risk, rate ratios or HRs because cardiovascular events and death were rare events. ${ }^{30}$ The analysis was stratified based on whether the results had considered the effect of potential confounders through adjustments or propensity-matched cohorts or not. In order to reduce the risk of bias from confounding so that we have a more reliable estimate of the independent effect of bleeding on prognosis, we appraised studies with multivariate adjustments or propensity-matched cohorts separately from studies with crude or unadjusted results. We used adjusted or propensity-matched risk estimates where available. For datasets reporting multiple time points, we took the earliest time point for the primary analysis. Any later time points were subsequently analysed in the separate Forest plots stratified according to timing of outcome assessment. Where there were multiple definitions of major bleeding we choose to use the results for TIMI major bleeding.

We planned for three analyses. The primary analysis was the pooled adjusted and unadjusted risk of mortality with and without major bleed. The secondary analysis 
was the unadjusted and adjusted risk of MACEs with and without major bleed. In a post hoc sensitivity analysis, we conducted a pooled analysis after excluding studies where it was clear that only some (and not all) of the participants had undergone PCI.

\section{Statistical heterogeneity}

We used the $\mathrm{I}^{2}$ statistic to assess statistical heterogeneity. $\mathrm{I}^{2}$ Values of $30-60 \%$ represent moderate levels of heterogeneity. ${ }^{31}$

\section{RESULTS}

\section{Study selection}

The process of selection of studies is shown in online supplementary figure $\mathrm{S} 1$. We retrieved 42 relevant studies of patients that underwent PCI (total number of participants 533 333), which evaluated the risk of adverse events with and without major bleeding. ${ }^{2} 811 \quad 14$ 17-23 2728 32-58 The number of participants in each study ranged from 352 to 280390 and the number of major bleeding events was 66277 (37 studies, 15.3\%). A total of 40 studies evaluated mortality as an outcome and 11 studies reported on MACEs.

\section{Description of studies included}

The study designs, date of study, country of origin and indication for PCI is shown in table 1 . There were 12 studies (149650 participants), which were post hoc analyses of randomised controlled trials and 1 matched observational study (280390 participants). Of the studies that reported number of centres, there were more multicentre studies than single centre studies $(\mathrm{n}=17$ and $\mathrm{n}=13)$. The age and gender of participants along with the antiplatelet and anticoagulant regimes used is shown in online supplementary table S1.

Table 2 shows the timing of major bleeding, definition of major bleeding used in the individual studies and the incidence of major bleeding in each study. While many of the studies did not report when assessment for major bleeding took place $(n=15), 15$ of the studies clearly stated that they evaluated in-hospital major bleeding. The definitions of major bleeding also varied among the included studies and formal definitions of major bleeding included TIMI, GUSTO, STEEPLE, HORIZON-AMI, CRUSADE, BARC and REPLACE-2 definitions. These definitions are summarised in online supplementary table S2. Follow-up of patients in the studies included in this analysis varied from $48 \mathrm{~h}$ to more than 3 years. The impact of major bleeding on clinical outcomes was adjusted for baseline covariates in 22 studies (490 699 participants) while in 20 studies (42 634 participants) only unadjusted outcomes were reported.

\section{Quality assessment}

Online supplementary table S3 shows the quality assessment for included studies. Ascertainment of bleeding and mortality varied from data collection from medical record reviews to prospective evaluation in trials where independent committees adjudicated bleeding and outcome events.

Major bleeding and risk of mortality at any time point

The impact of major bleeding on mortality outcomes was studied in 40 studies reporting outcomes in 525691 patients. In total, 66016 major bleeds were reported. Crude rates or risk estimates for mortality in individual studies are shown in online supplementary table S4. Mortality rate was 3595/62 036 (5.8\%, 28 studies) in patients who sustained a major bleed and 8937/370 522 (2.4\%, 28 studies) in patients not experiencing major bleeding complications.

Meta-analysis of these data demonstrated that the overall risk of mortality was significantly greater among patients who sustained major bleeding complications periprocedurally (figure 1). The risk estimate for mortality was significantly lower in the subgroup of studies, which adjusted for potential confounders (OR 3.31 (2.86 to 3.82 ), $\mathrm{I}^{2}=80 \%, 491565$ participants compared to the OR calculated in those studies that did not adjust for baseline covariates; OR 6.75 (4.99 to 9.12 ), $\mathrm{I}^{2}=62 \%$, 34126 participants).

\section{Major bleeding and risk of MACEs at any time point}

The impact of major bleeding on MACEs outcomes was studied in 13 studies reporting outcomes in 69843 patients. Crude rates or risk estimates for MACEs in individual studies are shown in tables 3 and 4; 757 major bleeds $(6.8 \%)$ were reported.

MACE rates were 295/1701 $(17.3 \%)$ in patients who sustained a major bleed and 2101/38 520 (5.4\%) in patients not experiencing major bleeding complications. The overall risk of MACEs was significantly higher among patients with major bleeds (figure 2). The risk of MACEs did not significantly differ in the subgroup of studies that adjusted for baseline covariates (OR 3.89 (3.26 to 4.64 ), $\mathrm{I}^{2}=42 \%, 25829$ participants as compared to those that did not adjust for baseline covariates OR 3.12 (2.32 to 4.19$), \mathrm{I}^{2}=52 \%, 101184$ participants).

Mortality with major bleeding at different follow-up durations The adjusted risk of mortality was significantly greater among patients with major bleeds at all time points evaluated (figure 3). For 30-day mortality, adjusted risk was OR 3.24 (2.73 to $3.84, \mathrm{I}^{2}=81 \%$, 11 studies, 403457 participants) and at 6 months, the OR remained elevated at 3.23 (2.92 to $3.57, \mathrm{I}^{2}=0 \%$, 4 studies, 50872 participants; table 3). At the 1-year time point, the risk of mortality was OR 3.64 (2.39 to $5.56, \mathrm{I}^{2}=89 \%, 9$ studies).

MACEs with bleeding at different follow-up durations

Analysis of the impact of major bleeding on MACEs outcomes at different time points was stratified into risk of 


\begin{tabular}{|c|c|c|c|c|c|}
\hline Study ID & Design & Date of study & $\begin{array}{l}\text { Number of } \\
\text { centres }\end{array}$ & Country & Inclusion criteria \\
\hline Amlani et al 32 & Prospective cohort & May 2003 to July 2007 & Single centre & Canada & Patients with STEMI \\
\hline Ariza-Sole et al ${ }^{33}$ & Prospective cohort & $\begin{array}{l}\text { October } 2009 \text { to April } \\
2012\end{array}$ & Single centre & Spain & Patients with STEMI \\
\hline Barthélémy et al ${ }^{20}$ & Prospective cohort & NA & Single centre & France & Patients in E-Paris Registry with STEMI and PCI \\
\hline Bertrand et al ${ }^{34}$ & $\begin{array}{l}\text { Post hoc analysis of } \\
\text { RCT }\end{array}$ & $\begin{array}{l}\text { October } 2003 \text { to April } \\
2005\end{array}$ & Single centre & Canada & Patients in EASY trial with $\mathrm{PCI}$ without STEMI \\
\hline Boden et al ${ }^{35}$ & Cohort & NA & NA & $\begin{array}{l}\text { The } \\
\text { Netherlands }\end{array}$ & Patients with STEMI and PCl \\
\hline Budaj et $a l^{36}$ & $\begin{array}{l}\text { Post hoc analysis of } \\
\text { RCT }\end{array}$ & NA & Multicentre & International & Patients with NSTEMI ACS. Note that only $64-79 \%$ had PCI \\
\hline Cayla et al ${ }^{18}$ & $\begin{array}{l}\text { Post hoc analysis of } \\
\text { RCT }\end{array}$ & NA & Multicentre & France & Patients in ABOARD trial with NSTEMI and PCI \\
\hline Cayla et $a^{37}$ & $\begin{array}{l}\text { Post hoc analysis of } \\
\text { RCT }\end{array}$ & NA & Multicentre & International & Patients in ATOLL study with STEMI and PCI \\
\hline Chhatriwalla et al ${ }^{14}$ & Matched cohort & 2004 to 2011 & Multicentre & USA & Patients in the CathPCI Registry \\
\hline Correia et al ${ }^{38}$ & Cohort & $\begin{array}{l}\text { August } 2007 \text { to } \\
\text { December } 2010\end{array}$ & Multicentre & Brazil & $\begin{array}{l}\text { Patients with ACS. Note that only } 76-90 \% \text { had coronary } \\
\text { angiogram }\end{array}$ \\
\hline Eikelboom et al ${ }^{28}$ & $\begin{array}{l}\text { Post hoc analysis of } \\
\text { RCTs }\end{array}$ & NA & Multicentre & International & $\begin{array}{l}\text { Patients in OASIS } 1 \text { and } 2 \text { and CURE who had ACS. Note } \\
\text { that only } 10-11 \% \text { had } \mathrm{PCI} / \text { stent/atherectomy }\end{array}$ \\
\hline Fuchs et al ${ }^{21}$ & Prospective cohort & $\begin{array}{l}\text { January } 2001 \text { to June } \\
2005\end{array}$ & Single centre & Israel & Patients with STEMI and PCl \\
\hline Gitt et al ${ }^{39}$ & Cohort & $\begin{array}{l}\text { October } 2006 \text { to } \\
\text { October } 2008\end{array}$ & Multicentre & International & $\begin{array}{l}\text { Patients with ACS. Note that only } 65-82 \% \text { had primary/rescue } \\
\mathrm{PCl}\end{array}$ \\
\hline Giugliano et $a^{8}$ & $\begin{array}{l}\text { Post hoc analysis of } \\
\text { RCT }\end{array}$ & NA & Multicentre & International & $\begin{array}{l}\text { Patients in ExTRACT-TIMI } 25 \text { trial where patients received } \\
\text { fibrinolysis }\end{array}$ \\
\hline Hermanides et al ${ }^{22}$ & Prospective cohort & $\begin{array}{l}\text { January } 1991 \text { to } \\
\text { December } 2004\end{array}$ & Single centre & $\begin{array}{l}\text { The } \\
\text { Netherlands }\end{array}$ & Patients with STEMI and PCI \\
\hline Kaul et al ${ }^{40}$ & $\begin{array}{l}\text { Post hoc analysis of } \\
\text { RCT }\end{array}$ & $\begin{array}{l}\text { May } 2004 \text { to August } \\
2008\end{array}$ & Multicentre & International & Patients with NSTEMI \\
\hline Kikkert et al ${ }^{41}$ & Prospective cohort & $\begin{array}{l}\text { January } 2003 \text { to July } \\
2008\end{array}$ & Single centre & $\begin{array}{l}\text { The } \\
\text { Netherlands }\end{array}$ & Patients with STEMI and PCI \\
\hline Kinnaird et al ${ }^{11}$ & Retrospective cohort & 1991 to 2000 & NA & USA & Patients who underwent $\mathrm{PCl}$ \\
\hline Le May et al ${ }^{42}$ & Cohort & May 2005 to July 2010 & NA & Canada & Patients with STEMI and PCI \\
\hline Lee et al ${ }^{43}$ & Prospective cohort & $\begin{array}{l}\text { February } 2003 \text { to March } \\
2006\end{array}$ & NA & Korea & Patients with $\mathrm{PCl}$ with DES \\
\hline Lemesle et al ${ }^{27}$ & Cohort & $\begin{array}{l}\text { January } 2000 \text { to } \\
\text { December } 2007\end{array}$ & Single centre & USA & Patients $\geq 80$ years of age with $\mathrm{PCl}$ \\
\hline Lindsey et al ${ }^{44}$ & Prospective cohort & $\begin{array}{l}\text { July } 2004 \text { to January } \\
2006\end{array}$ & Multicentre & USA & Patients in EVENT registry with $\mathrm{PCl}$ \\
\hline Lopes et al ${ }^{45}$ & Retrospective cohort & $\begin{array}{l}\text { November } 2001 \text { to } \\
\text { December } 2006\end{array}$ & Multicentre & USA & Patients in CRUSADES registry and subset with $\mathrm{PCl}$ \\
\hline
\end{tabular}


Table 1 Continued

\begin{tabular}{|c|c|c|c|c|c|}
\hline Study ID & Design & Date of study & $\begin{array}{l}\text { Number of } \\
\text { centres }\end{array}$ & Country & Inclusion criteria \\
\hline Matic et al ${ }^{46}$ & Cohort & $\begin{array}{l}\text { August } 2009 \text { to } \\
\text { December } 2012\end{array}$ & NA & Serbia & Patients with STEMI and PCI \\
\hline Matic et al ${ }^{47}$ & Cohort & $\begin{array}{l}\text { August } 2009 \text { to } \\
\text { December } 2010\end{array}$ & NA & Serbia & Patients with STEMI and PCI \\
\hline Matic et al ${ }^{48}$ & Prospective cohort & $\begin{array}{l}\text { August } 2009 \text { to } \\
\text { December } 2010\end{array}$ & NA & Serbia & Patients with STEMI and PCI \\
\hline Mehran et $a l^{2}$ & $\begin{array}{l}\text { Post hoc analysis of } \\
\text { RCTs }\end{array}$ & NA & Multicentre & International & $\begin{array}{l}\text { Patients in REPLACE-2, ACUITY and HORIZONS-AMI trial } \\
\text { with } \mathrm{PCl}\end{array}$ \\
\hline Montalescot et al ${ }^{25}$ & $\begin{array}{l}\text { Post hoc analysis of } \\
\text { RCT }\end{array}$ & $\begin{array}{l}\text { December } 2005 \text { to } \\
\text { December } 2006\end{array}$ & Multicentre & International & Patients in STEEPLE trial who underwent elective $\mathrm{PCl}$ \\
\hline Mrdovic et al ${ }^{49}$ & Cohort & $\begin{array}{l}\text { February } 2006 \text { to } \\
\text { December } 2009\end{array}$ & Single centre & Serbia & Patients with STEMI and PCI \\
\hline Musumeci et al ${ }^{50}$ & Cohort & $\begin{array}{l}\text { June } 2005 \text { to June } \\
2008\end{array}$ & Multicentre & Italy & Patients with $\mathrm{PCl}$ and DES \\
\hline Ndrepepa et al ${ }^{17}$ & $\begin{array}{l}\text { Post hoc analysis of } \\
\text { RCT }\end{array}$ & $\begin{array}{l}\text { September } 2005 \text { to } \\
\text { January } 2008\end{array}$ & NA & Germany & Patients in ISAR-REACT-3 trial with $\mathrm{PCl}$ \\
\hline Ndrepepa et al ${ }^{23}$ & $\begin{array}{l}\text { Post hoc analysis of } \\
\text { RCT }\end{array}$ & NA & NA & Germany & $\begin{array}{l}\text { Patients in ISAR-REACT, ISAR-SWEET, ISAR-SMART and } \\
\text { ISAR-REACT-2 trials with PCI }\end{array}$ \\
\hline Pierre-Louis et al $^{51}$ & Cohort & NA & NA & USA & Patients with $\mathrm{ACS}$ and $\mathrm{PCl}$ \\
\hline Pilgrim and Wenaweser 52 & Cohort & $\begin{array}{l}\text { May } 2002 \text { to December } \\
2005\end{array}$ & Single centre & Switzerland & Patients with $\mathrm{PCl}$ \\
\hline $\begin{array}{l}\text { Polanska-Skrzypczyk } \\
\text { et al }\end{array}$ & Prospective cohort & $\begin{array}{l}\text { February } 2001 \text { to } \\
\text { October } 2002\end{array}$ & Single centre & Poland & Patients with STEMI and PCI \\
\hline Poludasu et al $^{54}$ & Prospective cohort & July 2001 to May 2010 & Multicentre & USA & Patient with $\mathrm{PCl}$ \\
\hline Rao et al ${ }^{24}$ & $\begin{array}{l}\text { Post hoc analysis of } \\
\text { RCTs }\end{array}$ & NA & Multicentre & International & $\begin{array}{l}\text { Patients in GUSTO Ilb, PURSUIT and PARAGON A/B. Note } \\
\text { that only } 11-30 \% \text { had PCl }\end{array}$ \\
\hline Rossini et al ${ }^{55}$ & Cohort & NA & NA & USA & Patients with $\mathrm{PCl}$ and DES \\
\hline Urban et al ${ }^{56}$ & Prospective cohort & May 2006 to April 2008 & Multicentre & International & Patients in e-SELECT registry with $\mathrm{PCI}$ and DES \\
\hline Valente et al ${ }^{19}$ & Prospective cohort & $\begin{array}{l}\text { January } 2004 \text { to } \\
\text { December } 2008\end{array}$ & Single centre & Italy & $\begin{array}{l}\text { Patients in Intensive Cardiac Care Florence STEMI Registry } \\
\text { who had STEMI and PCl }\end{array}$ \\
\hline Yoon et al ${ }^{57}$ & Cohort & NA & NA & Korea & Patients in IRS DES registry with $\mathrm{PCI}$ and DES \\
\hline Zheng et al ${ }^{58}$ & Retrospective cohort & $\begin{array}{l}\text { January } 2004 \text { to } \\
\text { January } 2008\end{array}$ & Single centre & China & Patients with elective or urgent $\mathrm{PCl}$ \\
\hline
\end{tabular}


Table 2 Timing of bleeding, definition of major bleeding and follow-up

\begin{tabular}{|c|c|c|c|c|c|}
\hline Study ID & $\begin{array}{l}\text { Timing of } \\
\text { bleeding }\end{array}$ & Major/severe bleeding criteria & $\begin{array}{l}\text { Number of } \\
\text { participants in } \\
\text { bleeding group }\end{array}$ & $\begin{array}{l}\text { Number of } \\
\text { participants in } \\
\text { control group }\end{array}$ & Follow-up \\
\hline Amlani et $a^{32}$ & Within 30 days & $\begin{array}{l}\text { Haemoglobin drop } \geq 5 \mathrm{~g} / \mathrm{dL} \text {, intracranial haemorrhage, } \\
\text { bleeding requiring surgery or blood transfusion of at least } \\
2 \text { units }\end{array}$ & 152 (67 had PCl) & 1237 (566 had PCl) & 30 days \\
\hline Ariza-Sole et $a^{33}$ & In-hospital & CRUSADE & 33 & 1031 & $\begin{array}{l}\text { Mean follow-up } \\
344 \text { days }\end{array}$ \\
\hline Barthélémy et $\mathrm{al}^{20}$ & In-hospital & TIMI, GUSTO, STEEPLE & Total 671 & NA & 1 year \\
\hline Bertrand et al ${ }^{34}$ & Unclear & REPLACE-2 & 19 & 1329 & $\begin{array}{l}30 \text { days, } \\
6 \text { months, } \\
1 \text { year }\end{array}$ \\
\hline Boden et $a^{35}$ & In-hospital & CRUSADE & 203 & 762 & 1 year \\
\hline Budaj et al ${ }^{36}$ & Up to 180 days & ESSENCE & $\begin{array}{l}771 \text { (30 days), } 937 \\
\text { (180 days) }\end{array}$ & $\begin{array}{l}18851 \text { (30 days), } \\
18851 \text { (180 days) }\end{array}$ & 180 days \\
\hline Cayla et $a^{18}$ & 30 days & STEEPLE & 19 & 333 & 30 days \\
\hline Cayla et $a l^{37}$ & 30 days & STEEPLE & 42 & 868 & 30 days \\
\hline Chhatriwalla et al ${ }^{14}$ & In-hospital & CathPCI Registry definition & 56078 & 224312 & In-hospital \\
\hline Correia et al ${ }^{38}$ & In-hospital & BARC type 3 or 5 & 29 & 426 & In-hospital \\
\hline Eikelboom et al ${ }^{28}$ & Within 30 days & $\begin{array}{l}\text { Bleeding that was significantly disabling, bleeding requiring } \\
\text { transfusion of } \geq 2 \text { units of packed cells or bleeding that was } \\
\text { life threatening }\end{array}$ & 783 & 3363 & 30-day mortality \\
\hline Fuchs et $a l^{21}$ & Unclear & TIMI & 27 & 804 & $\begin{array}{l}30 \text { days and } \\
6 \text { months }\end{array}$ \\
\hline Gitt et $a l^{39}$ & In-hospital & Drop in haemoglobin of $>5 \mathrm{~g} / \mathrm{dL}$ or haematocrit of $>15 \%$ & 281 & 8451 & In-hospital \\
\hline Giugliano et $a l^{8}$ & Up to day 8 & TIMI & 309 & 20014 & 30 days \\
\hline Hermanides et al ${ }^{22}$ & $48 \mathrm{~h}$ & TIMI & 80 & 4371 & $\begin{array}{l}30 \text { days and } \\
1 \text { year }\end{array}$ \\
\hline Kaul et al ${ }^{40}$ & $\begin{array}{l}\text { Up to } 120 \mathrm{~h} \text { after } \\
\text { randomisation }\end{array}$ & GUSTO & 598 & 8808 & 30 days \\
\hline Kikkert et al ${ }^{41}$ & Unclear & TIMI & 35 & 331 & $1-30$ days \\
\hline Kinnaird et al ${ }^{11}$ & Unclear & TIMI & 588 & 8992 & In-hospital \\
\hline Le May et al ${ }^{42}$ & Unclear & TIMI & 91 & 1941 & 6 months \\
\hline Lee et al ${ }^{43}$ & Median 1366 days & STEEPLE & 148 & 3022 & $\begin{array}{l}\text { Median } \\
1366 \text { days }\end{array}$ \\
\hline Lemesle et al ${ }^{27}$ & In-hospital & $\begin{array}{l}\text { Decrease in haematocrit of } \geq 15 \% \text { and/or the occurrence of } \\
\text { a major haematoma/gastrointestinal bleeding/intracerebral } \\
\text { bleeding }\end{array}$ & 127 & 2639 & 6 months \\
\hline Lindsey et al ${ }^{44}$ & In-hospital & TIMI & 180 & 5781 & 1 year \\
\hline Lopes et al ${ }^{45}$ & In-hospital & CRUSADE & 3902 & 28993 & $\begin{array}{l}30 \text { days, } 1 \text { year, } \\
3 \text { year, }>3 \text { years }\end{array}$ \\
\hline Matic et al ${ }^{46}$ & Unclear & GUSTO & 32 & 738 & In-hospital \\
\hline Matic et al ${ }^{47}$ & Unclear & HORIZONS-AMI & 88 & 1154 & 30 day \\
\hline
\end{tabular}




\begin{tabular}{|c|c|c|c|c|c|}
\hline Study ID & $\begin{array}{l}\text { Timing of } \\
\text { bleeding }\end{array}$ & Major/severe bleeding criteria & $\begin{array}{l}\text { Number of } \\
\text { participants in } \\
\text { bleeding group }\end{array}$ & $\begin{array}{l}\text { Number of } \\
\text { participants in } \\
\text { control group }\end{array}$ & Follow-up \\
\hline Matic et al ${ }^{48}$ & Unclear & BARC & 114 & 1558 & 1 year \\
\hline Mehran et $a l^{2}$ & 30 days & TIMI & 267 & 17034 & Up to 1 year \\
\hline Montalescot et al ${ }^{25}$ & $48 \mathrm{~h}$ & STEEPLE & 3528 (total) & NA & 1 year \\
\hline Mrdovic et al ${ }^{49}$ & Unclear & TIMI & 22 & 2074 & $\begin{array}{l}30 \text { days and } \\
1 \text { year }\end{array}$ \\
\hline Musumeci et al ${ }^{50}$ & $\begin{array}{l}30 \text { days, } \\
12 \text { months, any } \\
\text { follow-up }\end{array}$ & TIMI & 52 & 1385 & 3 years \\
\hline Ndrepepa et $a^{17}$ & Unclear & REPLACE-2 & 555 & 4015 & 1 year \\
\hline Ndrepepa et $a l^{23}$ & In-hospital & TIMI, REPLACE-2, BARC & Total 12459 & NA & 30 days, 1 year \\
\hline Pierre-Louis et al ${ }^{51}$ & In-hospital & $\begin{array}{l}\text { Intracerebral or intraocular bleeding, clinical bleeding } \\
\text { requiring blood transfusion, clinical bleeding with a } \\
\text { reduction in haematocrit }>10 \text { points, retroperitoneal or } \\
\text { gastrointestinal bleeding, access site bleeding requiring } \\
\text { intervention, and } \geq 4 \mathrm{~cm} \text { diameter vascular access site } \\
\text { haematoma }\end{array}$ & 34 & 600 & In-hospital \\
\hline $\begin{array}{l}\text { Pilgrim and } \\
\text { Wenaweser }\end{array}$ & In-hospital & TIMI & 48 & 3787 & 30 days \\
\hline $\begin{array}{l}\text { Polanska-Skrzypczyk } \\
\text { et al }{ }^{53}\end{array}$ & In-hospital & TIMI & 40 & 1024 & 1 year \\
\hline Poludasu et al ${ }^{54}$ & Unclear & HORIZONS-AMI & 396 & 11595 & 2.3 years \\
\hline Rao et $a^{24}$ & Unclear & GUSTO & 2908 & 23544 & Up to 6 months \\
\hline Rossini et al ${ }^{55}$ & Unclear & TIMI & 57 & 1301 & 1 year \\
\hline Urban et $a l^{56}$ & Unclear & STEEPLE & Total 15147 & NA & 360 days \\
\hline Valente et al ${ }^{19}$ & In-hospital & TIMI, ACUITY & Total 991 & NA & In-ICCU \\
\hline Yoon et al ${ }^{57}$ & In-hospital & BARC type $2-5$ & 234 & 5932 & 2 years \\
\hline Zheng et $a l^{58}$ & Unclear & TIMI & 27 & 385 & 1 year \\
\hline
\end{tabular}




\begin{tabular}{|c|c|c|c|c|c|c|c|}
\hline Study or Subgroup & log[Odds Ratio] & SE & Weight & $\begin{array}{l}\text { Odds Ratio } \\
\text { IV, Random, } 95 \% \mathrm{Cl}\end{array}$ & & $\begin{array}{c}\text { Odds Ratio } \\
\text { IV, Random, } 95 \% \mathrm{Cl}\end{array}$ & \\
\hline \multicolumn{7}{|c|}{ 1.1.1 Unadjusted analysis } & \\
\hline Ariza-Sole 2013 & 1.93297 & 0.315633 & $2.4 \%$ & $6.91[3.72,12.83]$ & & & \\
\hline Barthelemey 2012 & 2.952825 & 0.521713 & $1.4 \%$ & $19.16[6.89,53.27]$ & & & \\
\hline Bertrand 2009 & 5.939803 & 1.567807 & $0.2 \%$ & $379.86[17.58,8206.22]$ & & & $\longrightarrow$ \\
\hline Boden 2012 & 1.396245 & 0.334086 & $2.3 \%$ & $4.04[2.10,7.78]$ & & & \\
\hline Cayla 2011 & 3.918005 & 0.817273 & $0.7 \%$ & $50.30[10.14,249.59]$ & & & \\
\hline Cayla 2012 & 1.871802 & 0.421276 & $1.8 \%$ & $6.50[2.85,14.84]$ & & & \\
\hline Fuchs 2009 & 1.449269 & 0.459527 & $1.6 \%$ & $4.26[1.73,10.48]$ & & & \\
\hline Kinnaird 2003 & 1.508512 & 0.174428 & $3.6 \%$ & $4.52[3.21,6.36]$ & & $\rightarrow$ & \\
\hline Lee 2009 & 1.818077 & 0.274042 & $2.7 \%$ & $6.16[3.60,10.54]$ & & & \\
\hline Matic 2010 & 1.261298 & 0.644726 & $1.0 \%$ & $3.53[1.00,12.49]$ & & & \\
\hline Matic 2011 & 0.746688 & 0.357863 & $2.2 \%$ & $2.11[1.05,4.25]$ & & & \\
\hline Mrdovic 2013 & 2.701361 & 0.446599 & $1.7 \%$ & $14.90[6.21,35.75]$ & & & \\
\hline Musumeci 2012 & 1.353255 & 0.385864 & $2.0 \%$ & $3.87[1.82,8.24]$ & & & \\
\hline Ndrepepa 2010 & 1.798404 & 0.320035 & $2.4 \%$ & $6.04[3.23,11.31]$ & & & \\
\hline Pierre 2010 & 4.864298 & 1.489577 & $0.2 \%$ & $129.58[6.99,2401.42]$ & & & \\
\hline Pilgrim 2010 & 2.200552 & 0.456042 & $1.6 \%$ & $9.03[3.69,22.07]$ & & & \\
\hline Rossini 2010 & 2.021548 & 0.347564 & $2.2 \%$ & $7.55[3.82,14.92]$ & & & \\
\hline Valente 2011 & 2.121063 & 0.328735 & $2.3 \%$ & $8.34[4.38,15.88]$ & & & \\
\hline Subtotal $(95 \% \mathrm{Cl})$ & & & $32.4 \%$ & $6.75[4.99,9.12]$ & & & \\
\hline \multirow{2}{*}{\multicolumn{8}{|c|}{ Heterogeneity: $\operatorname{Tau}^{2}=0.23 ; \mathrm{Chi}^{2}=44.66, \mathrm{df}=17(\mathrm{P}=0.0003) ; \mathrm{I}^{2}=62 \%$}} \\
\hline & & & & & & & \\
\hline \multicolumn{8}{|c|}{ 1.1.2 Adjusted analysis } \\
\hline Amlani 2010 & 1.131402 & 0.357863 & $2.2 \%$ & $3.10[1.54,6.25]$ & & & \\
\hline Budaj 2009 & 1.241269 & 0.145547 & $3.8 \%$ & $3.46[2.60,4.60]$ & & $\rightarrow$ & \\
\hline Chhatriwalla 2013 & 1.068153 & 0.024481 & $4.4 \%$ & $2.91[2.77,3.05]$ & & - & \\
\hline Correia 2012 & 1.205971 & 0.527799 & $1.3 \%$ & $3.34[1.19,9.40]$ & & & \\
\hline Eikelboom 2006 & 1.680828 & 0.153983 & $3.7 \%$ & $5.37[3.97,7.26]$ & & & \\
\hline Gitt 2010 & 0.779325 & 0.209011 & $3.3 \%$ & $2.18[1.45,3.28]$ & & - & \\
\hline Giugliano 2010 & 1.064711 & 0.103435 & $4.1 \%$ & $2.90[2.37,3.55]$ & & $\rightarrow$ & \\
\hline Hermanides 2010 & 1.252763 & 0.217727 & $3.2 \%$ & $3.50[2.28,5.36]$ & & $\rightarrow$ & \\
\hline Kaul 2013 & 1.388791 & 0.17187 & $3.6 \%$ & $4.01[2.86,5.62]$ & & $\rightarrow$ & \\
\hline Kikkert 2013 & 1.007958 & 0.258763 & $2.9 \%$ & $2.74[1.65,4.55]$ & & - & \\
\hline Le May 2011 & 1.497388 & 0.359071 & $2.1 \%$ & $4.47[2.21,9.04]$ & & & \\
\hline Lemesle 2009 & 1.098612 & 0.212477 & $3.2 \%$ & $3.00[1.98,4.55]$ & & $\rightarrow$ & \\
\hline Lindsey 2009 & 1.924249 & 0.36129 & $2.1 \%$ & $6.85[3.37,13.91]$ & & & \\
\hline Lopes 2012 & 0.693147 & 0.166889 & $3.6 \%$ & $2.00[1.44,2.77]$ & & $\rightarrow$ & \\
\hline Matic 2012 & 1.098612 & 0.293016 & $2.6 \%$ & $3.00[1.69,5.33]$ & & & \\
\hline Mehran 2011 & 1.435085 & 0.155373 & $3.7 \%$ & $4.20[3.10,5.70]$ & & $\rightarrow$ & \\
\hline Montalescot 2009 & 1.098612 & 0.518602 & $1.4 \%$ & $3.00[1.09,8.29]$ & & & \\
\hline Ndrepepa 2012 & 1.144223 & 0.159025 & $3.7 \%$ & $3.14[2.30,4.29]$ & & $\rightarrow$ & \\
\hline Polanska 2011 & 1.788421 & 0.389938 & $2.0 \%$ & $5.98[2.78,12.84]$ & & & \\
\hline Poludasu 2011 & 0.336472 & 0.13994 & $3.8 \%$ & $1.40[1.06,1.84]$ & & 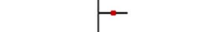 & \\
\hline Rao 2005 & 1.474763 & 0.074902 & $4.3 \%$ & $4.37[3.77,5.06]$ & & - & \\
\hline $\begin{array}{l}\text { Urban } 2011 \\
\text { Subtotal }(95 \% \mathrm{Cl})\end{array}$ & 1.791759 & 0.302455 & $\begin{array}{r}2.5 \% \\
67.6 \%\end{array}$ & $\begin{array}{r}6.00[3.32,10.85] \\
3.31[2.86,3.82]\end{array}$ & & $\diamond$ & \\
\hline \multicolumn{8}{|c|}{$\begin{array}{l}\text { Heterogeneity: } \text { Tau }^{2}=0.07 ; \mathrm{Chi}^{2}=102.70, \mathrm{df}=21(\mathrm{P}<0.00001) ; \mathrm{I}^{2}=80 \% \\
\text { Test for overall effect: } Z=16.32(\mathrm{P}<0.00001)\end{array}$} \\
\hline Total $(95 \% \mathrm{Cl})$ & & & $100.0 \%$ & $4.14[3.59,4.78]$ & & $\checkmark$ & \\
\hline \multicolumn{5}{|c|}{$\begin{array}{l}\text { Heterogeneity: } \operatorname{Tau}^{2}=0.12 ; \mathrm{Chi}^{2}=203.27, \mathrm{df}=3 \\
\text { Test for overall effect: } Z=19.44(P<0.00001)\end{array}$} & 0.01 & 1 & 10 \\
\hline
\end{tabular}

Table 3 Summary of risk of mortality and MACEs among patients with and without major bleed after percutaneous coronary intervention at different time points

\begin{tabular}{|c|c|c|c|c|c|}
\hline Duration & OR & $\mathrm{I}^{2}(\%)$ & $\begin{array}{l}\text { Number of } \\
\text { participants }\end{array}$ & $\begin{array}{l}\text { Number of events } \\
\text { in bleed group/total }\end{array}$ & $\begin{array}{l}\text { Number of events in } \\
\text { non-bleed group/total }\end{array}$ \\
\hline \multicolumn{6}{|l|}{ Adjusted risk of mortality } \\
\hline 30 days $^{8} 14242832363839404145$ & $3.24(2.73$ to 3.84$)$ & 81 & 403457 & $3307 / 59630$ & $7645 / 317375$ \\
\hline 6 months $^{24273642}$ & 3.23 (2.92 to 3.57$)$ & 0 & 50872 & $154 / 1028$ & $1119 / 20606$ \\
\hline 1 year $^{2} 22232544485356$ & $3.64(2.39$ to 5.56$)$ & 89 & 74637 & $160 / 2494$ & $677 / 36167$ \\
\hline \multicolumn{6}{|l|}{ Adjusted risk of MACEs } \\
\hline$<1$ year $^{243436}$ & 3.96 (3.26 to 4.81$)$ & 53 & 47422 & $175 / 790$ & $1204 / 20180$ \\
\hline$>1$ year $^{43}$ & $3.19(1.89$ to 5.38$)$ & NA & 3170 & NA & NA \\
\hline
\end{tabular}


Table 4 Summary of risk of mortality and MACEs among patients with and without major bleed after percutaneous coronary intervention with different definitions of bleeding

\begin{tabular}{|c|c|c|c|c|c|c|c|}
\hline \multicolumn{4}{|l|}{ Mortality } & \multicolumn{4}{|l|}{ MACEs } \\
\hline Definition of major bleed & OR & $I^{2}(\%)$ & $\begin{array}{l}\text { Number of } \\
\text { participants }\end{array}$ & Definition & OR & $I^{2}$ & $\begin{array}{l}\text { Number of } \\
\text { participants }\end{array}$ \\
\hline $\mathrm{BARC}^{233848}$ & $5.40(1.74$ to 16.74$)$ & 88 & 14550 & BARC & NA & NA & NA \\
\hline CRUSADE $^{33} 3545$ & 3.69 (1.68 to 8.12$)$ & 85 & 14055 & CRUSADE & NA & NA & NA \\
\hline GUSTO $^{24} 4046$ & 4.30 (3.76 to 4.92$)$ & 0 & 27222 & GUSTO $^{24}$ & 4.37 (3.78 to 5.07 ) & NA & 26452 \\
\hline HORIZON-AMI ${ }^{47} 54$ & 1.51 (1.11 to 2.05$)$ & 12 & 13233 & HORIZON-AMI & NA & NA & NA \\
\hline REPLACE $^{172334}$ & 6.69 (2.26 to 19.81$)$ & 84 & 17996 & REPLACE & NA & NA & NA \\
\hline STEEPLE $^{18} 25374356$ & 6.59 (3.89 to 11.16$)$ & 53 & 23107 & STEEPLE $^{43}$ & 3.19 (1.89 to 5.37$)$ & NA & 3170 \\
\hline 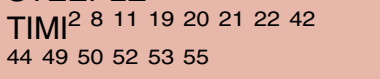 & $5.15(4.01$ to 6.61$)$ & 69 & 69449 & $\mathrm{TIMI}^{21}$ & 2.41 (1.42 to 4.35$)$ & NA & 1348 \\
\hline Other ${ }^{14} 272832363951$ & 3.34 (2.59 to 4.32 ) & 76 & 346670 & Other ${ }^{36}$ & 3.99 (3.3 to 4.82 ) & NA & 19622 \\
\hline
\end{tabular}

the greatest impact on mortality outcomes, while the ACUITY/HORIZONS-AMI had the least impact (OR 1.51 (1.11 to 2.05$), \mathrm{I}^{2}=12 \%, 1$ study, 13233 participants).

\section{Sensitivity analysis}

Sensitivity analysis excluding studies where not all participants had PCI did not significantly alter the pooled analysis for association between major bleeding and mortality (OR 3.16 (2.61 to 3.81 ), $\mathrm{I}^{2}=75 \%, 372449$ participants).

\section{DISCUSSION}

Advances in antithrombotic and antiplatelet therapy have improved outcomes following PCI through the reduction of ischaemic events although this has been at the expense of increased procedure-related bleeding complications. Our meta-analysis of 42 studies including over half a million patients confirms that major bleeding is independently associated with a threefold increase in mortality and MACEs outcomes, and that this increased mortality and MACEs risk observed following a major bleed is sustained for periods of over 1 year. We also show that major bleeding events as defined by different contemporary bleeding definitions have differential impacts on mortality outcomes, with 1.5-6.7-fold increases in mortality observed depending on the definition of major bleeding used.

There are a number of potential mechanisms that may underlie the relationship between major bleeding and adverse mortality outcomes. Patients that sustain major bleeding complications post PCI are more likely to be older, have renal failure, undergo PCI for STEMI/ NSTEMI presentations, present with haemodynamic compromise or have a history of heart failure ${ }^{259}$ that also

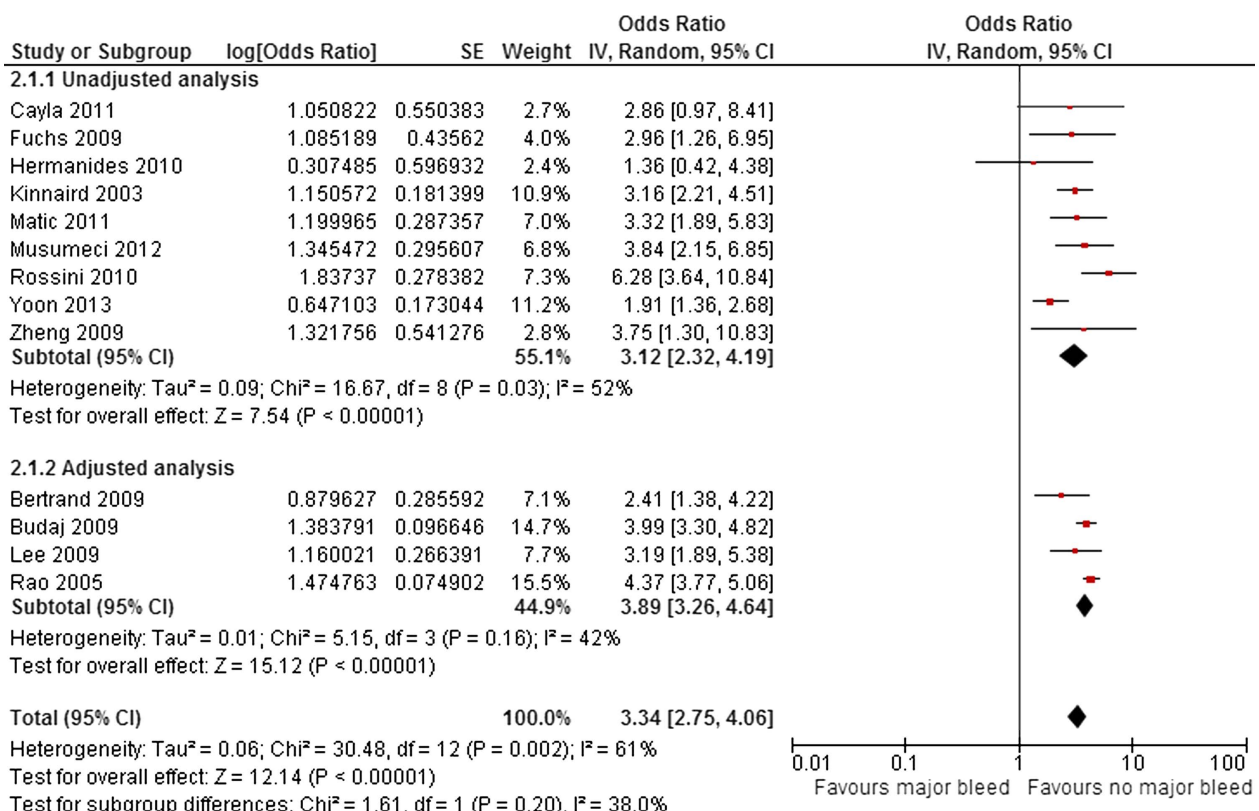

Figure 2 Adjusted risk of major adverse cardiovascular event with and without major bleed after percutaneous coronary intervention. 


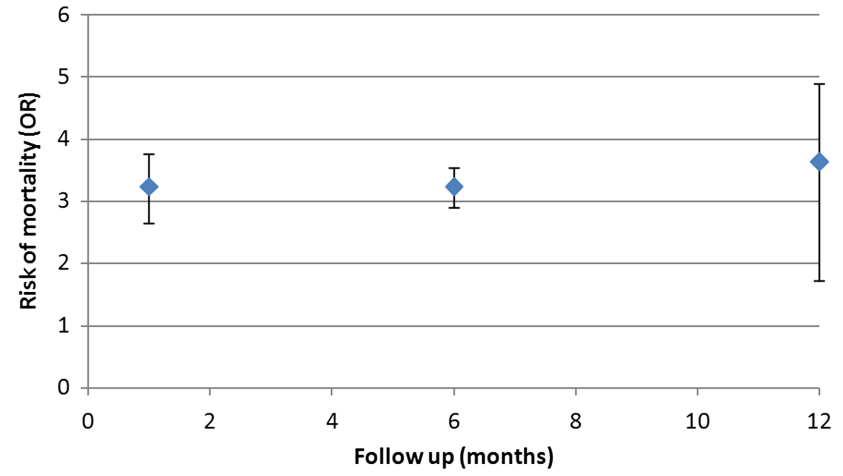

Figure 3 Risk of mortality with major bleed with different duration of follow-up.

independently predict adverse mortality outcomes themselves. Many previous studies ${ }^{17-21}$ that have reported on the prognostic impact of major bleeds have not accounted for differences in such baseline covariates that are themselves known to impact on mortality outcomes, which would overestimate the mortality risks associated with a major bleed. For example, in an analysis from the Global Registry of Acute Coronary Events (GRACE), major bleeding was no longer associated with 6-month mortality after adjustment for the known comorbidities with the authors concluding that comorbidities associated with major bleeding accounting for the higher rate of mortality in patients who bled. ${ }^{15}$ Consistent with this, we have observed that in meta-analysis of studies that do not adjust for baseline covariates, major bleeding is associated with a sixfold increased risk in mortality, but this decreases to a threefold independent increase in mortality once baseline covariates are adjusted for, suggesting that major bleeding is independently linked to mortality and MACEs outcomes.

The potential mechanisms by which a major bleed adversely impacts on clinical outcomes are numerous. Major bleeds such as an intracranial haemorrhage or severe blood loss as can occur in a gastrointestinal haemorrhage may result in mortality directly, although such bleeding events would not explain the persistent mortality risk observed for over a year following a major bleeding event in our meta-analysis. Major bleeds may necessitate the discontinuation of antiplatelet or antithrombotic medications that increase the risk for stent thrombosis, a strong independent predictor of mortality outcomes in the short and longer term $\left(>1\right.$ year). ${ }^{60}$ Furthermore, increased production of erythropoietin in response to anaemia that occurs following a major bleed may contribute to a prothrombotic systemic state beyond the acute phase through platelet activation and induction of plasminogen activator inhibitor-1 (PAI-1). ${ }^{61}{ }^{62}$ Treatment with erythropoietin has been associated with increased risk of thrombosis in critical care patients ${ }^{63}$ and increase in the composite endpoint of death, acute myocardial infarction, stroke and stent thrombosis in patients with STEMI. ${ }^{64}$ Blood transfusions have been linked to adverse shorter and longer term mortality ${ }^{65}$ and have been shown to predict 30-day mortality ${ }^{66}$ independently of bleeding and haematocrit. Adverse mortality outcomes associated with blood transfusions occur through a number of mechanisms including prothrombotic effects mediated through acute platelet release of CD40 ligand, ${ }^{67}$ platelet activation and induction of PAI- ${ }^{61}$ an inhibitor of endogenous fibrinolytic mechanisms. These increased risks of mortality also extend to non-red blood cell transfusions such as platelets or plasma/cryoprecipitate that may also be utilised following a periprocedural major bleed. ${ }^{68}$

Our observations of a differential impact of different major bleeding definitions on mortality outcomes is particularly pertinent suggesting that the choice of bleeding definition used has significantly influenced the outcome of previous studies, since the definition employed will influence the prevalence of reported bleeds as well as their prognostic impact. For example, in the RIVAL study ${ }^{69}$ non-CABG related major bleeding as defined by the study was not significantly different between the radial and femoral arms of the study $(\mathrm{OR}=0.73,95 \% \mathrm{CI}$ 0.43 to $1.23 ; \mathrm{p}=0.9$ ), while use of the ACUITY major bleeding criterion was associated with a statistically significant reduction in major bleeding in the radial arm of the study ( $\mathrm{OR}=0.43$, 95\% CI 0.32 to $0.57 ; \mathrm{p}<00001)$. Similarly, in the SYNERGY trial that used the GUSTO and TIMI major definition of bleeding, the enoxaparin arm was associated with a significantly higher rate of major bleeding as defined by TIMI major criteria but no difference in major bleeding as defined by the GUSTO major definition. Definitions that encompass less severe bleeding events (such as the ACUITY definition) are not as strongly linked to adverse events and therefore may not be a powerful means of evaluating bleeding avoidance strategies. In order to prove efficacy for bleeding avoidance the use of a more discriminative definition would be preferable.

Our meta-analysis has a number of potential limitations. First, an inherent limitation of any meta-analysis is that of publication bias; studies that show a neutral outcome in mortality are less likely to be published than those that show a positive outcome and thus tend to bias any meta-analysis of published data towards a more positive outcome. Second, studies included in this meta-analysis often used different antithrombotic and antiplatelet regimes for the PCI procedures undertaken for different indications, hence it is unclear whether the prognostic impact of a major bleed differs with different antiplatelet/anticoagulant combinations or whether it differs in the elective/acute coronary syndrome setting. Finally, our current analysis does not provide insight into whether the timing of the major bleed in relation to the index PCI procedure has a differential impact on mortality outcomes.

In conclusion, our meta-analysis of 42 studies including over half a million patients has revealed that major bleeding is independently associated with a threefold 
increase in mortality and MACEs outcomes, and that this increased mortality and MACEs risk observed following a major bleed is observed for periods of over 1 year. We also show that major bleeding events as defined by different contemporary bleeding definitions have differential impacts on mortality outcomes. Given the significant impact of major bleeding on mortality outcomes, formal bleeding risk assessment should be undertaken as part of the decision-making process for PCI procedures and bleeding avoidance strategies such as optimal pharmacotherapy and access site choice should be actively undertaken, particularly in those patients at highest baseline risk for bleeding complications.

\section{Author affiliations}

${ }^{1}$ Cardiovascular Institute, University of Manchester, Manchester, UK

${ }^{2}$ Department of Cardiology, Duke Clinical Research Institute, Duke University Medical Center, Durham, North Carolina, USA

${ }^{3}$ Division of Applied Health Sciences, School of Medicine \& Dentistry, University of Aberdeen, Aberdeen, Scotland, UK

${ }^{4}$ Department of Cardiology, University Hospital North Staffordshire, Stoke-onTrent, UK

${ }^{5}$ Department of Cardiology, Queen Elizabeth Hospital, Birmingham, UK ${ }^{6}$ Cardiothoracic Division, The James Cook University Hospital, Middlesbrough, UK

${ }^{7}$ Norwich Medical School, University of East Anglia, Norwich, UK

Contributors CSK, MAM and YKL contributed to planning, conduct and reporting of the work. SVR, PKM, BK, JN, PFL and MDB contributed to the interpretation of the findings and reporting of the work. MAM is the guarantor and was responsible for the overall content.

Competing interests None.

Provenance and peer review Not commissioned; externally peer reviewed.

Data sharing statement No additional data are available.

Open Access This is an Open Access article distributed in accordance with the Creative Commons Attribution Non Commercial (CC BY-NC 3.0) license, which permits others to distribute, remix, adapt, build upon this work noncommercially, and license their derivative works on different terms, provided the original work is properly cited and the use is non-commercial. See: http:// creativecommons.org/licenses/by-nc/3.0/

\section{REFERENCES}

1. Lincoff AM, Kleiman NS, Kereiakes DJ, et al. Long-term efficacy of bivalirudin and provisional glycoprotein IIb/llla blockade vs heparin and planned glycoprotein Ilb/llla blockade during percutaneous coronary revascularization: REPLACE-2 randomized trial. JAMA 2004;292:696-703.

2. Mehran R, Pocock S, Nikolsky E, et al. Impact of bleeding on mortality after percutaneous coronary intervention results from a patient-level pooled analysis of the REPLACE-2 (randomized evaluation of $\mathrm{PCl}$ linking angiomax to reduced clinical events), ACUITY (acute catheterization and urgent intervention triage strategy), and HORIZONS-AMI (harmonizing outcomes with revascularization and stents in acute myocardial infarction) trials. JACC Cardiovasc Interv 2011;4:654-64.

3. Subherwal S, Peterson ED, Dai D, et al. Temporal trends in and factors associated with bleeding complications among patients undergoing percutaneous coronary intervention: a report from the National Cardiovascular Data CathPCI Registry. J Am Coll Cardiol 2012;59:1861-9.

4. Mehta SR, Jolly SS, Cairns, et al. RIVAL Investigators. Effects of radial versus femoral artery access in patients with acute coronary syndromes with or without ST-segment elevation. J Am Coll Cardiol 2012;60:2490-9.

5. Mehran R, Pocock SJ, Stone GW, et al. Associations of major bleeding and myocardial infarction with the incidence and timing of mortality in patients presenting with non-ST-elevation acute coronary syndromes: a risk model from the ACUITY trial. Eur Heart $J$ 2009;30:1457-66.

6. Happe LE, Rao SV, Horblyuk R, et al. Consequences of major bleeding in hospitalized patients with non-ST segment elevation acute coronary syndromes receiving injectable anticoagulants. Curr Med Res Opin 2009;25:413-20.

7. Stone GW, Witzenbichler B, Guagliumi G, et al. Bivalirudin during primary $\mathrm{PCl}$ in acute myocardial infarction. $N$ Engl $\mathrm{J}$ Med 2008;358:2218-30.

8. Giugliano RP, Giraldez RR, Morrow DA, et al. Relationship between bleeding and outcomes in patients with ST-elevation myocardial infarction in the ExTRACT-TIMI 25 trial. Eur Heart J 2010;31:2103-10.

9. Rao SV, Mehran R. Evaluating the bite of the BARC. Circulation 2012;125:1344-6.

10. Mehran R, Rao SV, Bhatt DL, et al. Standardized bleeding definitions for cardiovascular clinical trials: a consensus report from the bleeding academic research consortium. Circulation 2011;123:2736-47.

11. Kinnaird TD, Stabile E, Mintz GS, et al. Incidence, predictors, and prognostic implications of bleeding and blood transfusion following percutaneous coronary interventions. Am J Cardiol 2003;92:930-5.

12. Manoukian SV, Feit F, Mehran R, et al. Impact of major bleeding on 30-day mortality and clinical outcomes in patients with acute coronary syndromes: an analysis from the ACUITY trial. J Am Coll Cardiol 2007:49:1362-8.

13. Rao SV, Eikelboom JA, Granger CB, et al. Bleeding and blood transfusion issues inpatients with non-ST-segment elevation acute coronary syndromes. Eur Heart J 2007;28:1193e204.

14. Chhatriwalla AK, Amin AP, Kennedy KF, et al. Association between bleeding events and in-hospital mortality after percutaneous coronary intervention. JAMA 2013;309:1022-9.

15. Spencer FA, Moscucci M, Granger CB, et al. Does comorbidity account for the excess mortality in patients with major bleeding in acute myocardial infarction? Circulation 2007;116:2793-801.

16. Berger PB, Manoukian SV. Bleeding is bad...isn't it? Circulation 2007;116:2776-8.

17. Ndrepepa G, Schulz S, Keta D, et al. Bleeding after percutaneous coronary intervention with bivalirudin or unfractionated heparin and one-year mortality. Am J Cardiol 2010;105:163-7.

18. Cayla G, Silvain J, Barthelemy O, et al. Trans-radial approach for catheterisation in non-ST segment elevation acute coronary syndrome: an analysis of major bleeding complications in the ABOARD Study. Heart 2011;97:887-91.

19. Valente S, Lazzeri C, Chiostri M, et al. STEMI patients-the more you bleed, the more you die: a comparison between classifications. Clin Cardiol 2011;34:90-6.

20. Barthélémy O, Silvain J, Brieger D, et al. Bleeding complications in primary percutaneous coronary intervention of ST-elevation myocardial infarction in a radial center. Catheter Cardiovasc Interv 2012;79:104-12.

21. Fuchs S, Kornowski R, Teplitsky I, et al. Major bleeding complicating contemporary primary percutaneous coronary interventions-incidence, predictors, and prognostic implications. Cardiovasc Revasc Med 2009;10:88-93.

22. Hermanides RS, Ottervanger JP, Dambrink JH, et al. Incidence, predictors and prognostic importance of bleeding after primary $\mathrm{PCI}$ fo ST-elevation myocardial infarction. Eurolntervention 2010;6:106-11.

23. Ndrepepa G, Schuster T, Hadamitzky M, et al. Validation of the bleeding academic research consortium definition of bleeding in patients with coronary artery disease undergoing percutaneous coronary intervention. Circulation 2012;125:1424-31.

24. Rao SV, O'Grady K, Pieper KS, et al. Impact of bleeding severity on clinical outcomes among patients with acute coronary syndromes. Am J Cardiol 2005;96:1200-6.

25. Montalescot G, Gallo R, White HD, et al. Enoxaparin versus unfractionated heparin in elective percutaneous coronary intervention 1-year results from the STEEPLE (SafeTy and efficacy of enoxaparin in percutaneous coronary intervention patients, an international randomized evaluation) trial. JACC Cardiovasc Interv 2009;2:1083-91.

26. Rao SV, O'Grady K, Pieper KS, et al. A comparison of the clinical impact of bleeding measured by two different classifications among patients with acute coronary syndromes. J Am Coll Cardiol 2006;47:809-16.

27. Lemesle G, Labriolle AD, Bonello L, et al. Impact of bivalirudin on in-hospital bleeding and six-month outcomes in octogenarians undergoing percutaneous coronary intervention. Cather Cardiovasc Interv 2009;74:428-35.

28. Eikelboom JW, Mehta SR, Anand SS, et al. Adverse impact of bleeding on prognosis in patients with acute coronary syndromes. Circulation 2006;114:774-82. 
29. Ioannidis JP, Trikalinos TA. The appropriateness of asymmetry test for publication bias in meta-analyses: a large survey. CMAJ 2007;176:1091-6.

30. Davies HT, Crombie IK, Tavakoli M. When odds ratios mislead? BMJ 1998;316:989-91.

31. Deeks JJ, Higgins JP, Altman DG. Analysing data and undertaking meta-analyses. In: Higgins JPT, Green S. eds. Cochrane handbook for systematic reviews of interventions. John Wiley \& Sons, 2008. http://handbook.cochrane.org/chapter_9/9_5_2_identifying_and_ measuring_heterogeneity.htm

32. Amlani S, Nadarajah T, Afzal R, et al. Mortality and morbidity following a major bleed in a registry population with acute ST elevation myocardial infarction. J Thromb Thrombolysis 2010;30:434-40.

33. Ariza-Sole A, Sanchez-Elvira G, Sanchez-Salado JC, et al. CRUSADE bleeding risk score validation for ST-segment-elevation myocardial infarction undergoing primary percutaneous coronary intervention. Thromb Res 2013;132:652-8.

34. Bertrand OF, Larose E, Rodés-Cabau J, et al. Incidence, predictors, and clinical impact of bleeding after transradial coronary stenting and maximal antiplatelet therapy. Am Heart J 2009;157: 164-9.

35. Boden $\mathrm{H}$, Velders $\mathrm{M}$, van der Hoeven, et al. The risk of in-hospital bleeding and long-term mortality in patients with ST elevation myocardial infarction treated with primary percutaneous coronary intervention. J Am Coll Cardiol 2012;60:B161-2.

36. Budaj A, Wikelboom JW, Mehta SR, et al. Improving clinical outcomes by reducing bleeding in patients with non-ST-elevation acute coronary syndrome. Eur Heart $J$ 2009;30:655-1.

37. Cayla G, Barthelemy O, Silvain J, et al. Anticoagulation, arterial access and bleeding in primary $\mathrm{PCl}$ : an analysis of the randomized ATOLL trial. Eur Heart $J$ 2012;33:462.

38. Correia LC, Sabino M, Brito M, et al. Causality analysis of the relationship between bleeding and lethality in acute coronary syndrome. Arq Bras Cardiol 2012;98:488-96.

39. Gitt AK, Bauer T, Hochadel MG, et al. Impact of radial arterial access for $\mathrm{PCl}$ in acute coronary syndromes on bleeding complications in clinical practice: results of the Euro Heart Survey PCl-registry. Eur Heart J 2010;31(Suppl 1):659.

40. Kaul P, Tanguay JF, Newby LK, et al. Association between bleeding and mortality among women and men with high-risk acute coronary syndromes: insights from the early versus delayed, provisional Eptifibatide in Acute Coronary Syndromes (EARLY ACS) trial. Am Heart J 2013;166:723-8.

41. Kikkert WJ, Zwindermann $\mathrm{AH}$, Vis MM, et al. Timing of mortality after severe bleeding and recurrent myocardial infarction in patients with ST-segment-elevation myocardial infarction. Circ Cardiovasc Interv 2013;6:391-8.

42. Le May M, MacDougall A, So D, et al. Impact of bleeding on mortality in patients referred for primary $\mathrm{PCl}$ in ST-elevation myocardial infarction. Can J Cardiol 27(5 Suppl 1):S219.

43. Lee JY, Kim YH, Kim WJ, et al. Long-term clinical impact of bleeding after percutaneous coronary intervention with drug-eluting stent in real world practice. J Am Coll Cardiol 2009;53:A45.

44. Lindsey JB, Marso SP, Pencina M, et al. Prognostic impact of periprocedural bleeding and myocardial infarction after percutaneous coronary intervention in unselected patients. Results from the EVENT (Evaluation of Drug-Eluting Stents and Ischemic Events) Registry. JACC Cardiovasc Interv 2009;2:1074-82.

45. Lopes RD, Subherwal S, Holmes DN, et al. The association of in-hospital major bleeding with short-, intermediate-, and long-term mortality among older patients with non-ST segment myocardial infarction. Eur Heart J 2012;33:2044-53.

46. Matic D, Mrdovic I, Stankovic G, et al. Incidence, predictors and prognostic implications of bleeding complicating contemporary primary percutaneous coronary interventions. Eur Heart J 2010;31 (Suppl 1):5.

47. Matic D, Stankovic G, Antonijevic N, et al. Prognostic implications of bleeding complicating STEMI treated with primary percutaneous coronary intervention. Eur Heart J 2011;32(Suppl 1):394.

48. Matic $D$, Milasinovic $D$, Asanin $M$, et al. Prognostic implications of bleeding measured by Bleeding Academic Research Consortium (BARC) categorization in patients treated with primary percutaneous coronary intervention. Eur Heart J 2012;33(Suppl 1):208-9.

49. Mrdovic I, Savic L, Asanin M, et al. Sex-related analysis of shortand long-term clinical outcomes and bleeding among patients treated with primary percutaneous coronary intervention: an evaluation of the RISK-PCI data. Can J Cardiol 2013;29: 1097-103.

50. Musumeci G, Rossini R, Lettieri C, et al. Prognostic implications of early and long-term bleeding events in patients on one-year dual antiplatelet therapy following drug-eluting stent implantation. Catheter Cardiovasc Interv 2012:80:395-405.

51. Pierre-Louis B, Aronow WS, Yoon JH, et al. Risk factors for major bleeding and for minor bleeding after percutaneous coronary intervention in 634 consecutive patients with acute coronary syndromes. Am J Ther 2010;17:e74-7

52. Pilgrim TM, Wenaweser P. Impact of bleeding on clinical outcome in patients undergoing PCI. Eur H J 2010;31(Suppl 1):658.

53. Polanska-Skrzypczyk M, Karcz M, Bekta P, et al. Major bleeding is a predictor of mid-term mortality in STEMI patients treated with primary $\mathrm{PCl}$. The ANIN myocardial infarction registry. Eur Heart J 2011;32 (Suppl 1):876-7.

54. Poludasu S, Baber U, Clark AE, et al. Impact of major bleeding on long term mortality in patients with and without anemia undergoing percutaneous coronary intervention with bivalirudin. TCT Abstracts JACC 2011;58(20 Suppl B):B17.

55. Rossini R, Musumeci G, Lettieri C, et al. Long-term bleeding events in patients with prolonged dual antiplatelet therapy after DES implantation. Eur Heart J 2010;31(Suppl 1):167.

56. Urban P, Abizaid A, Banning A, et al. Stent thrombosis and bleeding complications after implantation of sirolimus-eluting coronary stents in an unselected worldwide population: a report from the e-SELECT (Multi-Center Post-Market Surveillance) registry. J Am Coll Cardiol 2011;57:1445-54.

57. Yoon $\mathrm{YH}$, Kim YH, Park KM, et al. Impact of in-hospital bleeding on major adverse cardiac events and stroke using bleeding academic research consortium classification in patients who undergoing percutaneous coronary intervention. Am J Cardiol 2013;111 (Suppl 7):91B

58. Zheng HC, Q Zhang Q, Zhang RY, et al. Clinical outcomes of patients with major bleeding after primary coronary intervention for acute ST-segment elevation myocardial infarction. J Interv Radiol 2009;18:808-11.

59. Mehta SK, Frutkin AD, Lindsey JB, et al. Bleeding in patients undergoing percutaneous coronary intervention: the development of a clinical risk algorithm from the National Cardiovascular Data Registry. Circ Cardiovasc Interv 2009;2:222-9.

60. Dangas GD, Claessen BE, Mehran R, et al. Clinical outcomes following stent thrombosis occurring in-hospital versus out-of-hospital: results from the HORIZONS-AMI (Harmonizing Outcomes with Revascularization and Stents in Acute Myocardia Infarction) trial. J Am Coll Cardiol 2012;59:1752-9.

61. Doyle BJ, Rihal CS, Gastineau DA, et al. Bleeding blood transfusion and increased mortality after percutaneous coronary intervention. J Am Coll Cardiol 2009;53:2019-27.

62. Smith KJ, Bleyer AJ, Little WC, et al. The cardiovascular effects of erythropoietin. Cardiovasc Res 2003;59:538-48.

63. Corwin HL, Gettinger A, Fabian TC, et al. Efficacy and safety of epoetin alfa in critically ill patients. N Engl J Med 2007;357:965-76.

64. Najjar SS, Rao SV, Melloni C, et al. Intravenous erythropoietin in patients with ST-segment elevation myocardial infarction: REVEAL: a randomized controlled trial. JAMA 2011;305:1863-72.

65. Chase AJ, Fretz EB, Warburton WP, et al. Association of the arterial access site at angioplasty with transfusion and mortality: the M.O.R. T.A.L study (Mortality benefit Of Reduced Transfusion after percutaneous coronary intervention via the Arm or Leg). Heart 2008;94:1019-25.

66. Rao SV, Jollis JG, Harrington RA, et al. Relationship of blood transfusion and clinical outcomes in patients with acute coronary syndromes. JAMA 2004;292:1555-62.

67. Yacoub D, Hachem A, Théorêt JF, et al. Enhanced levels of soluble CD40 ligand exacerbate platelet aggregation and thrombus formation through a CD40-dependent tumor necrosis factor receptor-associated factor-2/Rac1/p38 mitogen-activated protein kinase signaling pathway. Arterioscler Thromb Vasc Biol 2010;30:2424-33.

68. Robinson SD, Janssen C, Fretz EB, et al. Non-red blood cell transfusion as a risk factor for mortality following percutaneous coronary intervention. Int J Cardiol 2012;157:169-73.

69. Jolly SS, Yusuf S, Cairns J, et al. Radial versus femoral access for coronary angiography and intervention in patients with acute coronary syndromes (RIVAL): a randomised, parallel group, multicentre trial. Lancet 2011;377:1409-20. 